\title{
EFFICIENT ESTIMATION UNDER PRIVACY RESTRICTIONS IN THE DISCLOSURE PROBLEM
}

\author{
Willem ALBERS \\ Department of Applied Mathematics, Twente University of Technologv, P.O. Box 217, 7500 AE Enschede, The Netherlands
}

Received October 1982

Revised August 1983

\begin{abstract}
In the disclosure problem already collected data are disclosed only to such extent that the individual privacy is protected to at least a prescribed level. For this problem estimators are introducted which are both simple and efficient.
\end{abstract}

Keywords: disclosure problem, efficient estimation, privacy protection.

\section{Introduction}

In this note we consider the so-called disclosure problem, which was discussed earlier by Warner (1971). Certain data have already been collected, for example by some government agency, and the point is how to disclose these data to interested investigators in such a way that the individual privacy is protected. To be more precise, we shall be concerned with the following situation here: Suppose we have at our disposal a sample $\left(X_{1}, \ldots, X_{N}\right)$ from some random variable (rv) $X$ and someone is interested in estimating $\mu=E X$. The problem then is to construct an unbiased estimator $T_{N}$ for $\mu$ based on our sample in such a way that the disclosure of $T_{N}$ will violate the individual privacy only up to a prescribed level according to some suitable measure.

To arrive at such a measure, we follow the approaches used by Leysieffer and Warner (1976) and Loynes (1976) for the closely related subject of randomized response procedures. Since this will typically cover all applications, we shall assume in what follows that all (conditional) distributions that occur are either discrete or continuous and we will denote the corresponding point probabilities or densities by $P_{X}, P_{T \mid X=x}, P_{X \mid T=t}$, etc. Now each function $R$ such that $\inf _{x} R(x)>1$ can be used to specify an upper bound on the privacy-risk for each individual as follows: require that for all $x$ and $t$ and $1 \leqslant k \leqslant N$

$P_{X_{k} \mid T_{N}-t}(x) \leqslant R(x) p_{X_{k}}(x)$.

Hence no outcome should be revealing to such extent that the a posteriori density of the true state differs by more than a prescribed factor from the corresponding a priori density. Since each individual has to be protected against the worst that can happen, this has to hold for all possible values of $t$ and $k$. This condition is intuitively attractive and moreover, it is equivalent to the bound of Leysieffer and Warner (1976), for the special case where $N=1$ and $X_{1}$ is dichotomous.

We shall restrict attention to constant $R$ here. In view of the definition (1.1), a region like $2 \leqslant R$ $\leqslant 10$ might be suitable for most applications.

Hence we shall now try to find a good estimator $T_{N}$ among the ones that satisfy (1.1). Note that in doing so we are basically faced with the dilemma that giving more information means less privacy but better estimators.

However, not every piece of information in $\left(X_{1}, \ldots, X_{N}\right)$ serves to improve the estimator. Hence we should refrain from revealing such information since it will only decrease the privacy without giving anything in return. For example, whenever $S_{N}=\sum_{i=1}^{N} X_{i}$ is a complete sufficient statistic for $\left(X_{1}, \ldots, X_{N}\right)$ with respect to $\mu=E X$, which occurs 
among others for the dichotomous and the normal case, we should at most reveal $S_{N}$ and certainly neglect the remaining information.

Note that $S_{N}$ itself may still be too revealing. If for example $X$ is known to satisfy $0 \leqslant X \leqslant d$ and the information is supplied that $S_{N}=N d$, then clearly all $X_{i}$ have to equal $d$. Now this example is not only very simple but also quite extreme and therefore it may be useful to give a second example. Let $P(X=1)=1-P(X=0)=\mu$, then

$P_{X_{1} \mid S_{*}=j}(1) / p_{X_{1}}(1)=\frac{j}{N \mu}$.

Observe that this ratio is large if $\mu$ is small compared to $j / N$. Since $\mu$ is not known and in typical applications involving embarrassing questions will indeed be quite small, we see that $S_{N}$ can also be too revealing for quite ordinary looking values $j$. However, $S_{N}$ will only be revealing if its outcome $j$ differs substantially from the expected value $N \mu$.

The case of bounded $X$ will be dealt with in Section 2. It turns out that here estimators based on $S_{N}$ indeed provide a completely satisfactory solution. In Section 3 we show that by using censoring the approach of Section 2 can be extended to the unbounded case.

\section{Bounded case: Sample mean}

In this section we shall suppose that $X$ has bounded support. To be specific we shall assume that for some known positive constant $d$ and for some possibly unknown $x_{0}$

$P\left(\left|X-x_{0}\right| \leqslant d / 2\right)=1$.

Since for bounded $X$ the most prominent candidate for estimation of $\mu$ certainly is $\bar{X}_{N}=S_{N} / N$, where $S_{N}=\sum_{i=1}^{N} X_{i}$, it seems reasonable to examine first the possibility $T_{N}+B_{N}\left(S_{N}\right) / N$, where $B_{N}$ is some known random function. As we already concluded in (1.2), the simple nonrandom choice $B_{N}(s)=s$ will not do. To see more clearly what goes wrong here, we note that $(1.1)$ is equivalent to

$P_{T_{N} \mid X_{h}=x}(t) \leqslant R(x) p_{T_{N}}(t)$.

Since

$P_{T_{v} \mid X_{k}=x}(t)=P_{S_{N} \mid X_{k}=x}(N t)=p_{S_{N-1}}(N t-x)$ for all $k$, while $P_{T_{i}}(t)=E p_{S_{N-1}}(N t-X)$, it follows that in this case (1.1) reduces for fixed $R$ to

$P_{S_{X-1}}(N t-x) \leqslant R E p_{S_{\text {S. }}}(N t-X)$,

for all $x$ and $t$. Now the asymptotic normality of $S_{v-1}$ will ensure that (2.2) holds for the (possibly quite small) central part of its distribution, but for extreme $t$ the condition can easily be violated (cf. once more example (1.2) with typically small $\mu$.)

A simple and quite satisfactory alternative $\bar{X}_{N}$ is presented in the following lemma.

Lemma 2.1. Let $Z$ be a ro independent of $\left(X_{1}, \ldots, X_{N}\right)$ and with density $\frac{1}{2} R^{-|x|} \log R$. Then condition (1.1) is satisfied for the following unbiased estimator of $\mu$

$T_{N}=\left(S_{N}+d Z\right) / N$,

with variance $\sigma^{2} / \mathrm{N}+2 \mathrm{~d}^{2} /\left(\mathrm{N}^{2} \log ^{2} \mathrm{R}\right)$.

Proof. For $T_{N}$ as in (2.3), condition (1.1) reduces to

$$
\begin{aligned}
& E p_{Z}\left(\left\{N t-S_{N-1}-x\right\} / d\right) \\
& \quad \leqslant R E p_{Z}\left(\left\{N T-S_{N}\right\} / d\right),
\end{aligned}
$$

for all $x$ and $t$. From (2.1) it follows that $S_{N-1}+x$ and $S_{N}$ never differ by more than an amount $d$, and hence the arguments of $p_{Z}$ on both sides of the inequality in (2.4) never differ by more than 1 . But $Z$ has been selected such that

$P_{Z}\left(z_{1}\right) \leqslant R p_{Z}\left(z_{2}\right)$,

for all $\left|z_{1}-z_{2}\right| \leqslant 1$. Hence (2.4), and thus (1.1), holds. The assertion about the variance of $T_{N}$ is immediate.

Remark 1. As concerns the application of the procedure, note that $Z$ is distributed as

$\operatorname{sign}(2 U-1) \log |2 U-1| / \log R$

where $U$ is a uniform $\mathrm{rv}$ on $(0,1)$.

Remark 2. The solution above applies to both continuous ánd discrete cases. However, if one finds it a bit awkward to use a continuous procedure in case of a discrete $X$, one might prefer the following discrete version, applicable to lattice rv's with span $h d$. Replace the double exponential $Z$ 
from $(2,3)$ by a rv $Z^{(h)}=W_{1}^{(h)}-W_{2}^{(h)}$, where $W_{1}^{(h)}$ and $W_{z}^{(h)}$ are independent identically distributed geometric rv's with parameter $R^{-h}$. Then

$\operatorname{var} Z^{(h)}=2 h^{2} R^{-h} /\left(R^{-h}-1\right)^{2}$,

which is decreasing in $h$. Moreover,

$\lim _{h \downarrow 0} \operatorname{var} Z^{(h)}=\operatorname{var} Z$.

Lemma 2.1 shows that the desired privacy protection can indeed be achieved with an asymptotically negligible variance increase. In fact, the deficiency $f_{N}$ of $T_{N}$ with respect to $S_{N}$ in the sense of Hodges and Lehmann (1970), which simply is the additional number of observations required by $T_{N}$ to attain the same variance as $S_{N}$, clearly tends to a finite limit $f$ which equals

$f=2 d^{2} /\left(\sigma^{2} \log ^{2} R\right)$.

The following suggest that not much can be gained by considering more complicated $T_{N}=$ $B_{N}\left(S_{N}\right) / N$ than the very simple choice from (2.3). Since $B_{N}$ is independent of $S_{N}$, we are in fact always dealing with a real parameter family $\left\{B_{N}(\theta)\right\}$. In analogy to $(2.5)$, this family should satisfy

$p_{B_{N}\left(\theta_{1}\right)}(N t) \leqslant R p_{B_{N}\left(\theta_{2}\right)}(N t), \quad\left|\theta_{1}-\theta_{2}\right| \leqslant d$.

It may be reasonable to assume that $E B_{N}(\theta)=\theta$. This has to hold whenever $S_{N}$ is a complete sufficient statistic. The objective is then to select $\left\{B_{N}(\theta)\right\}$ under these conditions such that var $T_{N}$ is small. Let $\tau^{2}(\theta)$ denote var $B_{N}(\theta)$, then

$\operatorname{var} T_{N}=\sigma^{2} / N+E \tau^{2}\left(S_{N}\right) / N^{2}$.

Since the distribution of $S_{N}$ is unknown, it follows that $\sup _{\theta} \tau^{2}(\theta)$ should be small.

Under these circumstances it is easy to verify that we can essentially restrict attention to $\left\{B_{N}(\theta)\right\}$ for which $p_{B_{N}(\theta)}(t)=\tilde{p}(t-\theta)$ for some fixed density $\tilde{p}$ with $\int t \tilde{p}(t) \mathrm{d} t=0$. It is also readily verified that $\tilde{p}$ can be assumed to be symmetric. Moreover, it seems reasonable to require that $\tilde{p}$ is nonincreasing on $(0, \infty)$. From these observations and (2.7) it then follows that $\tilde{p}(t) \geqslant \tilde{p}(t-d) / R$ for $t \geqslant d$. But is is easy to check that equality has to hold here almost surely, since otherwise a new density $p^{*}$ can be constructed which is better than $\tilde{p}$. The conclusion therefore is that it suffices to consider $B_{N}(\theta)$ of the form

$B_{N}(\theta)=\theta+d Z, \quad Z=V(W+Y)$,

where $V, W, Y,\left(X_{1}, \ldots, X_{N}\right)$ are independent, $P(V=1)=P(V=-1)=\frac{1}{2}, \quad P(W=k)=(R-$ 1) $/ R^{k+1}, k=0,1, \ldots$, while $Y$ is a continuous $\mathrm{rv}$ on $(0,1)$ such that $\sup p_{Y}(t)=R \inf p_{Y}(\mathrm{t})$.

The simple and smooth choice for $Z$ in Lemma 2.1 clearly satisfies (2.8). A small improvement still is possible, but the best $Z$ of the form (2.8) depends on $R$ in a relatively complicated way, so we will not bother to find it explicitly. We just mention as a second simple choice for which (2.8) holds $Z=W_{1}-W_{2}+U$, where $W_{1}$ and $W_{2}$ are independent copies of $W$ from (2.8) (cf. Remark $2)$, and $U$ is uniform on $\left(-\frac{1}{2}, \frac{1}{2}\right)$. Here var $Z=$ $2 R /(R-1)^{2}+\frac{1}{12}$, which differs very little from $2 / \log ^{2} R$ on the region of interest (e.g. for $R=2$ it equals 4.08 , compared to $2 / \log ^{2} 2=4.16$, while for $R=10$ we find 0.33 , compared to $2 / \log ^{2} 10=0.38$ ).

As an example, consider the dichotomous case mentioned before, with $P(X=1)=1-P(X=0)$ $=\mu$. Let $N=100, R=2$ and suppose $\mu$ lies just below 0.07 . Then, according to (1.2), $S_{N}$ is too revealing if its outcome exceeds 13 , which happens in about $1 \%$ of the cases. The additional variance of the estimator from (2.3) in this case equals 4.16.10 $0^{-4}$ and the asymptotic deficiency $f$ from (2.6) equals about 64 . Note that for $\mu$ in general $f$ equals $4.16 /\{\mu(1-\mu)\}$. Hence, if no protection is needed, which is the case when $\mu$ is not close to 0 or $1, f$ is considerably smaller, as should be the case.

\section{Unbounded case: Censoring}

In this section we shall briefly consider what happens if we drop the assumption (2.1) that $X$ has bounded support. This clearly invalidates the solution from the previous section. Now a highly unlikely value of $\bar{X}_{N}=S_{N} / N$ can be due entirely to one single $X_{k}$. One remedy is to replace $\bar{x}_{N}$, which is not robust against outliers, by an estimator in which the influence of the individual observations can be controlled. below we shall consider two possible approaches, both based on censoring.

The first approach uses type I censoring: let $\tilde{x}_{0}$ 
be a preliminary guess of $\mu$ and let $d_{N}, N=1,2, \ldots$ be a nondecreasing sequence of positive constants, which will typically tend to infinity as $N \rightarrow \infty$. Then replace $S_{N}$ by $\tilde{S}_{N}=\sum_{i=1}^{N} \tilde{X}_{i}$, where

$$
\tilde{X}_{i}= \begin{cases}X_{i} & \text { if }\left|X_{i}-\tilde{x}_{0}\right| \leqslant \frac{1}{2} d_{N}, \\ \frac{1}{2} d_{N} \operatorname{sign}\left(X_{i}-\tilde{x}_{0}\right) & \text { otherwise. }\end{cases}
$$

Clearly, the influence of $X_{k}$ on $\tilde{S}_{N}$ is at most $d_{N}$, and we can duplicate the approach from the previous section, with the following result.

Lemma 3.1. Let $Z$ be as in Lemma 2.1. Then condition (1.1) is satisfied if we replace $T_{N}$ by

$\tilde{T}_{N}=\left(\tilde{S}_{N}+d_{N} Z\right) / N$,

for which $E\left(\tilde{T}_{N}-\mu\right)^{2}=(\mu-\tilde{\mu})^{2}+\tilde{\sigma}^{2} / N+$ $2 d_{N}^{2} /\left(N^{2} \log ^{2} R\right)$, where $\tilde{\mu}=E X$ and $\tilde{\sigma}^{2}=\operatorname{var} \tilde{X}$. Moreover, suppose that $E g(|X|)<\infty$ for some $g$ on $(0, \infty)$ such that $g(x) / x^{2}$ is nondecreasing and let $h$ be the inverse of $g$. Then, for $d_{N}=h(N)$ and for each choice $\tilde{x}_{0}$

$E\left(\tilde{T}_{N}-\mu\right)^{2}=\sigma^{2} / N+\mathrm{O}\left(N^{-2} h^{2}(N)\right)$.

Proof. The first assertion is proved in exactly the same way as in Lemma 2.1. The expression for the mean squared error is obvious. To prove (3.3) we observe that, for $k=1,2$,

$$
\begin{aligned}
& E|X|^{k} I_{\mid d_{N} / 2, \infty},(|X|) \\
& \quad \leqslant\left(d_{N} / 2\right)^{k} g^{-1}\left(d_{N} / 2\right) E g(|X|) I_{\left[d_{N} / 2, \infty\right.},(|X|) \\
& =\mathrm{O}\left(d_{N}^{k} g^{-1}\left(d_{N}\right)\right),
\end{aligned}
$$

from which it follows that

$$
\begin{aligned}
& E\left(\tilde{T}_{N}-\mu\right)^{2}-\sigma^{2} / N \\
& \quad=\mathrm{O}\left(d_{N}^{2} g^{-2}\left(d_{N}\right)+N^{-1} d_{N}^{2} g^{-1}\left(d_{N}\right)+N^{-2} d_{N}^{2}\right),
\end{aligned}
$$

which is minimal for $d_{N}$ such that $g\left(d_{N}\right)=N$, i.e. for $d_{N}=h(N)$.

The lemma shows that even in the unbounded case guaranteed privacy protection is possible. The drawbacks are of course that $I_{N}$ is not translation invariant and only asymptotically unbiased, while the deficiency $f_{N}$ no longer has a finite limit as in (2.7), but now is of order $N^{-1} h^{2}(N)$.

In an attempt to remove these disadvantages, we also considered type II censoring. It turns out that trimmed means are successful is this respect. However, in this case an even more serious drawback occurs: the desired privacy protection is no longer provided with certainty, but merely with large probability. In view of this, we shall not consider this possibility any further.

\section{References}

Bickel, P.J. and E.L. Lehmann (1975), Descriptive statistics for nonparametric models, II: Location, Ann Statist. 3, 1045-1069.

Hodges, J.L., Jr. and E.L. Lehmann (1970), Deficiency, Ann. Math. Statist. 41, 783-801.

Huber, P.J. (1981), Robust Statistics, Wiley, New York.

Leysieffer, F.W. and S.L. Warner (1976), Respondent jeopardy and optimal designs in randomized response models, $J$. Amer. Statist. Ass. 71, 649-656.

Loynes, R.M. (1976), Asymptotically optimal randomized response procedures, J. Amer. Statist. Ass. 71, 924-928.

Warner, S.L. (1965), Randomized response: A survey technique for eliminating evasive answer bias, J. Amer. Statist. Ass. 60, 63-69.

Warner, S.L. (1971), The linear randomized response model, $J$. Amer. Statist. Ass. 66, 884-888. 